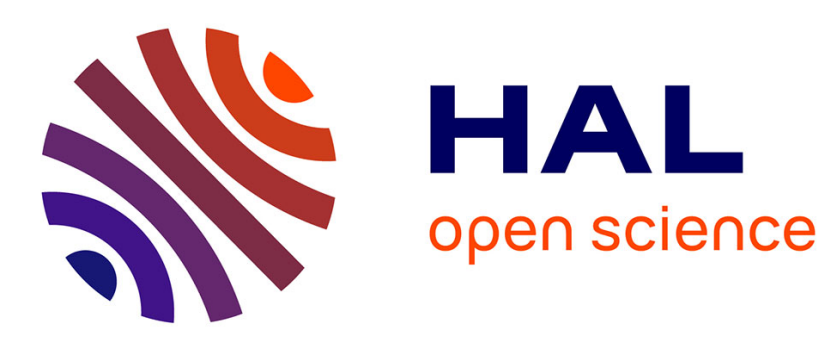

\title{
Several Forms of Fuzzy Analogical Reasoning
}

Bernadette Bouchon-Meunier, Jannick Delechamp, Christophe Marsala, Maria Rifqi

\section{To cite this version:}

Bernadette Bouchon-Meunier, Jannick Delechamp, Christophe Marsala, Maria Rifqi. Several Forms of Fuzzy Analogical Reasoning. 6th IEEE International Conference on Fuzzy Systems, Fuzz'IEEE'97, Jul 1997, Barcelona, Spain. pp.45-50, 10.1109/FUZZY.1997.616342 . hal-01072157

\section{HAL Id: hal-01072157 https://hal.inria.fr/hal-01072157}

Submitted on 7 Oct 2014

HAL is a multi-disciplinary open access archive for the deposit and dissemination of scientific research documents, whether they are published or not. The documents may come from teaching and research institutions in France or abroad, or from public or private research centers.
L'archive ouverte pluridisciplinaire HAL, est destinée au dépôt et à la diffusion de documents scientifiques de niveau recherche, publiés ou non, émanant des établissements d'enseignement et de recherche français ou étrangers, des laboratoires publics ou privés. 


\title{
Several Forms of Fuzzy Analogical Reasoning
}

\author{
B. Bouchon-Meunier*, J. Delechamp*o, C. Marsala*, M. Rifqi* \\ * LIP6, UPMC, Case 169 \\ 4 place Jussieu \\ 75252 Paris Cédex 05, France \\ \{bouchon, marsala, rifqi\}@laforia.ibp.fr \\ ${ }^{\circ} \mathrm{LCPC}$ \\ 58 boulevard Lefebvre \\ 75015 Paris, France \\ delecham@inrets.fr
}

\begin{abstract}
We present a general framework representing analogy, on the basis of a link between variables and measures of comparison between values of variables. This analogical scheme is proven to represent a common description of several forms of reasoning used in fuzzy control or in the management of knowledge-based systems, such as deductive reasoning, inductive reasoning or prototypical reasoning, gradual reasoning.
\end{abstract}

\section{Introduction}

Analogy is a natural means of drawing a conclusion in human reasoning. In artificial intelligence, analogy is also an explored domain, analogical reasoning and case-based reasoning have extensiveley been studied. In both approaches, the definition of resemblances is crucial and very often given in a prior way, but there exist very few studies on adapting an existing solution to a new piece of information.

Analogical reasoning has been formalized in a fuzzy set based approach in various directions [6][18], providing solutions for this adapting phase. Approximate reasoning has been presented by L.A. Zadeh as a method of automatic reasoning as close as possible to human reasoning [22]. Then, it seems natural that there is some relationship between approximate reasoning and analogy, which is at the root of most human reasoning processes. In this paper, we give a general analogical scheme which allows to regard several reasoning methods in a common framework.

\section{Analogical Scheme}

We consider two variables $X$ and $Y$, which may be simple or compound, defined on universes $X$ and $Y$. Let us denote by $F(X)$ and $F(Y)$ respective sets of fuzzy sets of $X$ and $Y$, which are either the respective sets $[0,1]^{X}$ and $[0,1]^{Y}$ of all fuzzy sets of $X$ and $Y$, or subsets of $[0,1]^{X}$ and $[0,1]^{Y}$.
For a given relation $\beta$ on $[0,1]^{\mathrm{X}} \times[0,1]^{\mathrm{Y}}$ and two relations $\mathrm{R}$ on $[0,1]^{\mathrm{X}} \times[0,1]^{\mathrm{X}}$ and $\mathrm{S}$ on $[0,1]^{\mathrm{Y}} \times[0,1]^{\mathrm{Y}}$, an analogical scheme is a function $\Re_{\beta R S}: F(X) \times F(Y) \times[0,1]^{X} \rightarrow[0,1]^{Y}$ satisfying : $\forall \mathrm{B} \in \mathrm{F}(\mathrm{X})$ and $\forall \mathrm{C} \in \mathrm{F}(\mathbb{Y})$ such that $\mathrm{B} \beta \mathrm{C}$,

$\forall \mathrm{B}^{\prime} \in[0,1]^{\mathrm{X}}$ such that $\mathrm{BRB}^{\prime}$,

(i) $\mathrm{C}=\Re_{\beta R S}(\mathrm{~B}, \mathrm{C}, \mathrm{B})$

(ii) $C^{\prime}=\Re_{\beta R S}\left(B, C, B^{\prime}\right)$ satisfies $\left(B^{\prime} \beta C^{\prime}\right.$ and $\left.C S C^{\prime}\right)$

(Figure 1)

We can interprete this scheme as follows, as soon as $\beta$, $R$ and $S$ are defined properly: if $B$ and $C$ are known to be linked by $\beta$, and if $B^{\prime}$ resembles $B$, we are able to find $C^{\prime}$ such that $B^{\prime}$ and $C^{\prime}$ are linked by $\beta$ and $C^{\prime}$ resembles $C$.

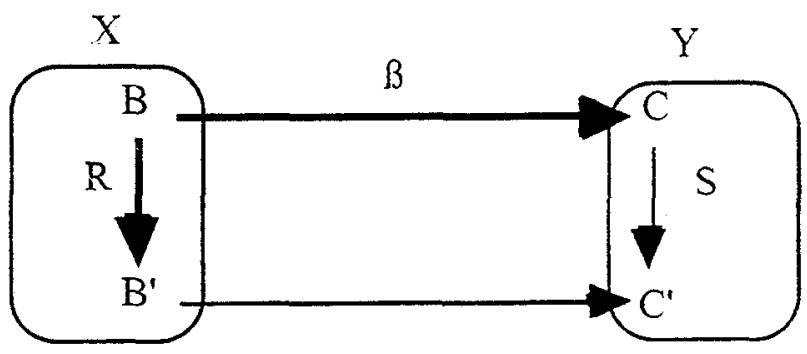

Figure 1. Analogical scheme

For instance, $B$ is a characterization of the attribute $X$ of an object and $Y$ is the class of this object and the link $\beta$ says that if, for an object, $\mathrm{X}$ is characterized by $\mathrm{B}$ then its class $Y$ is $C$. Then, if another object corresponds to a characterization $B^{\prime}$ of $X$ not very far from $B$, then the class we must assign to this new object is not very far from C.

Depending on the choice we make for $\beta, R, S$, the general definition of an analogical scheme yields various types of reasoning used in artificial intelligence.

3. Analogical Scheme for Inductive and Prototype-Based Reasoning 
Let us suppose that we are given a population of objects $\mathrm{E}$, characterized by values of a compound variable $\mathrm{X}=\left(\mathrm{X}_{1}, \ldots \mathrm{X}_{\mathrm{m}}\right)$, where $\mathrm{X}_{1}, \ldots \mathrm{X}_{\mathrm{m}}$ are elementary attributes, and a class $Y$, defined on $Y=\left\{y_{j} / j \in J\right.$. We suppose that, for a finite subset of $E$, used as a training set, values of $X$ and $Y$ are known. For other objects of $E$, the only value of $\mathrm{X}$ is known and the value of $\mathrm{Y}$ is to be determined.

For instance, in the well-known iris database of Fisher, objects are described by means of four attributes (the sepal length $X_{1}$, the sepal width $X_{2}$, the petal length $X_{3}$ and the petal width $X_{4}$ ) and they are associated with a class $Y$ referring to a type of iris plant (setosa, versicolor or virginica). We have $Y=$ \{Iris-Setosa, Iris-Versicolor, IrisVirginica $\}$. Such database could be downloaded from the ftp site of the University of California at Irvine, (ftp://ftp.ics.uci.edu/pub/machine-learning-databases).

Inductive learning consists in constructing a tree of attributes from the training set, in such a way that a path from the root of the tree to a leaf can be expressed as an ifthen rule $\left(R_{j}\right)$ for any $j \in J$. Prototype-based reasoning uses the training set to identify prototypical values of the attributes in each class $\left\{y_{j}\right\}$. Then, the membership of elements of $E$ to $y_{j}$ can be regarded as the satisfiability of a rule $\left(R_{j}\right)$ for any $j \in J$.

Examples of rules we can obtain for the iris database are the following:

(R1): if (petal-width is Small) then Iris-Setosa

(R2): if (petal-length is Medium) or ((petal-length is Long) and (petal-width is Small)) then Iris-Versicolor

(R3): if (petal-length is Long) and (petal-width is High) then Iris-Virginica

In both cases, we can use a fuzzy set based knowledge representation, allowing to avoid too strict limits of the values of attributes for a given class, and also to use in the same system either numerical (" $25 \mathrm{~km}$ ") or symbolic ("far") values of a given attribute.

We present briefly the learning phase in inductive learning and in prototype-based reasoning, then we exhibit their common analogical scheme for the classification or decision-making phase. We use the frameworks introduced in [4] and [16].

\subsection{Fuzzy inductive learning}

The aim of inductive learning is to find general rules enabling us to classify any object of $E$, i.e. to generalize the knowledge obtained by the observation of objects of $E$ in the training set to determine the value of $Y$ for any objects of $E$, when their only value of $X$ is known. The common inductive learning method is based on the construction of a decision tree from the training set. A decision tree is composed by three kinds of elements: nodes, edges and leaves. A node is associated with a question on the values of an attribute $X_{i}$ and each edge going out of a node is associated with a particular value (or modality) of $\mathrm{X}_{\mathrm{i}}$. A leaf, which is a terminal node, is labeled with a modality $y_{j}$ of the class $Y$. A path is composed of nodes linked by edges and ends in a leaf.

To build a decision tree is equivalent to choose an efficient order on the questions to ask on the values of the attributes for an object in order to determine its class. Usually, a question related to an attribute is selected by means of a measure of discrimination from the set of all possible questions. An example of such a measure is Shannon's measure of entropy, used for instance in the most common algorithm of construction of decision trees, the ID3 algorithm [13]. The modalities of the chosen attribute split the training set into subsets of objects. On each subset, another question regarding an attribute is selected until all the objects of the subset pertain to a single class.

Classical decision trees correspond to symbolic trees, built from training sets where all the attributes take their values in a finite set. To handle other existing kind of attributes, such as numerical attributes or numericalsymbolic attributes, new methods are introduced, either to construct decision trees or to use them in a generalization process [12]. When considering that the symbolic values of a numerical-symbolic attribute are fuzzy modalities on the numerical universe of its values, particular methods from fuzzy set theory to treat these values enable to take into account this kind of attributes. These methods enable to build fuzzy decision trees [14], [19], [11], [20], [1], [4]. Most of these methods are based on the ID3 algorithm and use particular techniques to take into account the imprecision in the data. Differences between them lie essentially in the choice of a new measure of discrimination to use during the construction of a fuzzy decision tree and in the discretization method to construct the fuzzy modalities associated with edges. The chosen measure takes into account the discriminating power of an attribute and, also, fuzzy modalities for the numericalsymbolic attributes.

The use of such fuzzy decision trees to classify new objects of $E$ is based on an extension of the classic method of utilization of decision tree. In addition to that, it enables to associate more than one class to an object, each class weighted by a membership degree [4]. A decision tree (either basic or fuzzy) is considered as a rule base. All the questions associated with nodes of a path of the tree are aggregated into a set of questions by means of the AND operator, and all the set of paths ending in a leaf labeled by the same value $y_{j}$ of the class $Y$ are aggregated into a rule $R_{j}$, by means of the OR operator. Thus, a set of if-then rules $R_{j}$ is obtained, the premises of these rules are the 
aggregated sets of questions and their conclusion is the corresponding value $y_{j}$ of the class $Y$.

\subsection{Fuzzy prototype-based reasoning}

The aim of prototype-based reasoning is to construct a fuzzy prototype for each class. A fuzzy prototype synthesizes a class and enables to generate a set of objects because of the information it contains [21]. The power of description of a prototype can be used for a classification process.

The notion of prototype is linked to the notion of typicality [17], [21]. The construction of a prototype needs to determine the typicality of each value appearing in a learning database.

We consider that the degree of typicality of an object depends positively on its total resemblance to other objects of its class (internal resemblance) and on its total dissimilarity to objects of other classes (external dissimilarity) [15].

We suppose that there exists a partition given on the set of objects $E$ composed by crisp classes $y_{j}$. The typicality of the value $B$ of an attribute $X_{i}$ of an object of the class $\mathrm{y}_{\mathrm{j}}$ is computed as follows:

Step 1. Compute the resemblance $r\left(B, B_{1}\right)$ between $B$ and the value $B_{1}$ of the attribute $X_{i}$ for any example of the same class $y_{j}$. The global resemblance $R(B)$ relative to the set of values of $X$ present in examples, is obtained in aggregating the degrees $\mathrm{r}\left(\mathrm{B}, \mathrm{B}_{1}\right)$ computed as above described.

Step 2. Compute the dissimilarity $d\left(B, B_{1}\right)$ between $B$ and the value $B_{1}$ of the attribute $X$ for any example of class $y_{k}$ different from $y_{j}$. The total dissimilarity $D(B)$ relative to the set of values of $X_{i}$ present in examples, is obtained in aggregating the degrees $\mathrm{d}\left(\mathrm{B}, \mathrm{B}_{1}\right)$ computed as above described.

Step 3. The aggregation of this two values, $R(B)$ et $D(B)$, gives the typicality $T(B)$ of $B$, according to the attribute $X_{i}$ for the class $y_{j}$.

The fuzzy prototype is composed by the most typical values for each attribute of a considered class. This means that a fuzzy prototype is a virtual object described by means of the same attributes as those pertaining to the learning database.

A prototype can be considered as a rule describing a class [3]. The classification process is based on a comparison between the object to be classified and a prototype. The question is: does the new object satisfy a prototype? The computed degrees of satisfiability are aggregated in order to obtain a total degree of satisfiability of a new object for a prototype.

\subsection{Analogical scheme}

The if-then rules obtained by both methods are of the following form :

$$
\begin{aligned}
& \left(R_{j}\right): \text { if }\left(X_{i_{1}(1)}^{j} \text { is } B_{i_{1}(1)}^{j} \text { and } X_{i_{1}(2)}^{j} \text { is } B_{i_{1}(2)}^{j} \text { and } \ldots\right) \\
& \text { or } \ldots\left(X_{i_{n}(1)}^{j} \text { is } B_{i_{n}(1)}^{j} \text { and } X_{i_{n}(2)}^{j} \text { is } B_{i_{n}(2)}^{j}\right. \text { and ...) } \\
& \text { then } Y \text { is } C_{j}, \\
& \text { with } C_{j}=\left\{y_{j}\right\}, i_{p}(1)<i_{p}(2)<\ldots, \text { and } \\
& \left\{X_{i_{1}(1)}^{j}, X_{i_{1}(2)}^{j} \ldots\right\} \subseteq\left\{X_{1}, \ldots X_{m}\right\} \ldots \\
& \left(X_{i_{n}(1)}^{j}, X_{i_{n}(2)}^{j} \ldots\right) \subseteq\left\{X_{1}, \ldots X_{m}\right\}
\end{aligned}
$$

Let us consider $F(\mathbb{Y})=\left\{C_{j} / j \in J\right\}$ and $F(X)$ the set of all corresponding values $\left(B_{1}, \ldots B_{m}\right)$ of $X$ appearing in all the rules, such that $B_{i_{p}(1)}=B_{i_{p}(1)}^{j}, B_{i_{p}(2)}=B_{i_{p}(2)}^{j}$ $\ldots$ for $1 \leq p \leq n, 1 \leq j \leq m$, with $B_{u}$ equal to $X$ if no index $i_{p(1)}, i_{p(2)}, \ldots$ is equal to $u$, which means that $X_{u}$ has no influence on the identification of $Y$ in this case $(1 \leq \mathrm{u} \leq \mathrm{m})$.

The link $\beta$ is defined by :

$\left(B_{1}, \ldots B_{m}\right) \beta C \Leftrightarrow$ i) or ii) holds with :

i) $\exists$ an object in $E$ with value of $X$ equal to $\left(B_{1}, \ldots B_{m}\right)$ and value of $Y$ equal to $C$

ii) $\exists R_{j}$ such that $B_{i_{p}(1)}=B_{i_{p}(1)}^{j}, B_{i_{p}(2)}=B_{i_{p}(2)}^{j}, \ldots$

with $\mathrm{p}=1$ or $\ldots \mathrm{n}$.

Let us consider a measure of satisfiability $\mathrm{r}$ [5], for instance defined by :

$r\left(B, B^{\prime}\right)=\min _{k} \frac{\int_{X} \min \left(B_{k}(x), B_{k}^{\prime}(x)\right) d x}{\int_{X} B_{k}(x) d x}$,

where the $\min$ operator can be replaced by another aggregation operator.

We define the relation $R$ as :

$$
\begin{aligned}
& \forall \mathrm{B}=\left(\mathrm{B}_{1}, \ldots \mathrm{B}_{\mathrm{m}}\right) \in[0,1]^{\mathrm{X}} \quad \forall \mathrm{B}^{\prime}=\left(\mathrm{B}_{1}^{\prime}, \ldots \mathrm{B}_{\mathrm{m}}^{\prime}\right) \in[0,1]^{\mathrm{X}} \\
& \mathrm{BRB}^{\prime} \Leftrightarrow \mathrm{r}\left(\mathrm{B}, \mathrm{B}^{\prime}\right)>0
\end{aligned}
$$

In the case where a crisp decision or class must be identified, the relation $S$ is the identity :

$\mathrm{CSC}^{\prime} \Leftrightarrow \mathrm{C}=\mathrm{C}^{\prime}$

and we consider the function

$\Re_{\beta R S}: \mathrm{F}(\mathrm{X}) \times \mathrm{F}(\mathrm{Y}) \times[0,1]^{\mathrm{X}} \rightarrow[0,1]^{\mathrm{Y}}$

defined as follows:

$\Re_{\beta R S}\left(B, C, B^{\prime}\right)=C \Leftrightarrow r\left(B, B^{\prime}\right)=\underset{B^{\prime \prime} \in F(X)}{1} r\left(B^{\prime \prime}, B^{\prime}\right)$

where $\perp$ is an aggregation operator, generally the $t$ conorm max:mum.

Then it is easy to prove that $\mathfrak{R}_{\beta \mathrm{RS}}$ is an analogical scheme. 
This means, that for an object of $E$ with a description $B^{\prime}$ of $X$, we look for the description $B$ of $X$ available in the rules which is as close as possible to $B^{\prime}$, and we choose its associated class $\mathrm{C}$ to assign to this object.

In the case where we can provide a fuzzy decision or class as a result of the reasoning, the relation $\mathrm{S}$ is defined from a resemblance relation $s$ by

$\forall \mathrm{C} \in[0,1]^{\mathrm{Y}} \quad \forall \mathrm{C}^{\prime} \in[0,1]^{\mathrm{Y}} \quad \mathrm{CSC}^{\prime} \Leftrightarrow \mathrm{s}\left(\mathrm{C}, \mathrm{C}^{\prime}\right)>0$, with for instance :

$s\left(C, C^{\prime}\right)=\sup _{j} \min \left(C\left(y_{j}\right), C^{\prime}\left(y_{j}\right)\right)$

We consider now the function $\mathfrak{R}_{\beta R S}$ defined as follows :

$\forall \mathrm{j} \in \mathrm{J} \Re_{\text {RRS }}\left(\mathrm{B}, \mathrm{C}_{\mathrm{j}}, \mathrm{B}^{\prime}\right)=\mathrm{C}^{\prime} \Leftrightarrow$

$r\left(B, B^{\prime}\right)=\frac{1}{\left\{B^{\prime \prime} / B^{\prime \prime} \in F(X), B^{\prime \prime} B C_{j}\right\}} r\left(B^{\prime \prime}, B^{\prime}\right)$

and $C^{\prime}\left(y_{j}\right)=r\left(B, B^{\prime}\right)$

It can be proven that $\Re_{\beta R S}$ is an analogical scheme.

This means that, for an object of $E$ with a description $B^{\prime}$ of $X$, we look for a fuzzy class $Y$, i.e. a fuzzy subset $\mathrm{C}^{\prime}$ of $\mathrm{Y}$ obtained by assigning to each possible decision $Y_{j}$ in $Y$ a membership degree equal to the satisfiability of the description $B$ of $X$ available in the rules which has the greatest satisfiability measure with $B^{\prime}$, and such that $B$ is linked with $C_{j}=\left\{y_{j}\right\}$ (i.e. $B \beta C_{j}$ ).

\section{Analogical scheme for deductive reasoning}

We consider $F(X)$ and $F(Y)$ respective finite subsets of $[0,1]^{X}$ and $[0,1]^{Y}$ and $\left(R_{j}\right)$ a base of rules of the form "if $X$ is $B_{j}$ then $Y$ is $C_{j}$ ", with $B_{j}$ in $F(X)$ and $C_{j}$ in $F(Y)$, $j \in J$, with $B_{j}$ and $C_{j}$ normalized fuzzy sets. With the same example as in section 3 , we can have :

if (petal-width is Small) then Iris-Setosa

The link $\beta$ is defined by:

$B \beta C \Leftrightarrow$ i) or ii) holds with :

i) $\exists$ an object in $E$ with value of $X$ equal to $B$ and value of $Y$ equal to $\mathrm{C}$

ii) $\exists R_{j}$ such that $B=B_{j}$ and $C=C_{j}$

The relations $R$ and $S$ are defined from measures of satisfiability $\mathrm{r}$ and $\mathrm{s}$ respectively defined on $[0,1]^{\mathrm{X}}$ and $[0,1]^{Y}$, in such a way that there exist two thresholds $\rho$ and $\sigma$ in $[0,1]$ such that

$\forall \mathrm{B} \forall \mathrm{B}^{\prime} \in[0,1]^{\mathrm{X}} \quad \mathrm{BRB}^{\prime} \Leftrightarrow \mathrm{r}\left(\mathrm{B}, \mathrm{B}^{\prime}\right) \geq \rho$

$\forall \mathrm{C} \quad \forall \mathrm{C}^{\prime} \in[0,1]^{\mathrm{X}} \quad \mathrm{CSC}^{\prime} \Leftrightarrow \mathrm{s}\left(\mathrm{C}, \mathrm{C}^{\prime}\right) \geq \sigma$.

The function $\Re_{\beta R S}$ is defined as follows :
$\Re_{\beta R S}\left(B, C, B^{\prime}\right)=C^{\prime} \Leftrightarrow C^{\prime}$ is obtained from $\mathrm{B}, \mathrm{C}, \mathrm{B}^{\prime}$ by means of the so-called compositional rule of inference.

$\forall y \in \mathbb{Y} C^{\prime}(y)=\sup _{x \in \mathbb{X}} T\left(B^{\prime}(x), I(x, y)\right)$,

where $I(x, y)$ is a fuzzy implication and $T$ is a $t$-norm.

It can be proven that $\mathfrak{R}_{\beta R S}$ is an analogical scheme with the following choices for the relations :

1) Measures of satisfiability :

$$
\begin{aligned}
& r\left(B, B^{\prime}\right)=\inf _{x \in X} \min \left(1-B^{\prime}(x)+B(x), 1\right) \\
& s\left(C, C^{\prime}\right)=\inf _{y \in Y} \min \left(1-C^{\prime}(y)+C(y), 1\right)
\end{aligned}
$$

$R$ and $S$ are defined by any equal thresholds $\rho=\sigma$ in $[0,1]$, for the following fuzzy implications :

$\mathrm{I}(\mathrm{x}, \mathrm{y})=1-\mathrm{B}(\mathrm{x})+\mathrm{B}(\mathrm{x}) \mathrm{C}(\mathrm{y})$ (Reichenbach)

$I(x, y)=\max (1-B(x), C(y))$ (Kleene - Dienes)

$I(x, y)=\min (1-B(x)+C(y), 1)$ (Lukasiewicz)

We choose the Lukasiewicz $t$-norm

$T(a, b)=\max (a+b-1,0)$.

2) Measures of satisfiability

$\mathrm{r}\left(\mathrm{B}, \mathrm{B}^{\prime}\right)=1-\sup _{\{\mathrm{x} \in \mathrm{X} / \mathrm{B}(\mathrm{x})=0\}} \mathrm{B}^{\prime}(\mathrm{x})$
$\mathrm{s}\left(\mathrm{C}, \mathrm{C}^{\prime}\right)=1-\sup _{\{\mathrm{y} \in \mathrm{Y} / \mathrm{C}(\mathrm{y})=0\}} \mathrm{C}^{\prime}(\mathrm{y})$

$R$ and $S$ are defined by any equal thresholds $\rho=\sigma$ in $[0,1]$, for the following fuzzy implications :

$I(x, y)=\left\{\begin{array}{l}1 \text { if } B(x) \leq C(y) \\ 0 \text { otherwise }\end{array}\right.$ (Rescher-Gaines)

$I(x, y)=\left\{\begin{array}{l}1 \text { if } B(x) \leq C(y) \\ C(y) \text { otherwise }\end{array}\right.$ (Brouwer $-G$ del )

$I(x, y)=\left\{\begin{array}{l}\min \left(\frac{C(y)}{B(x)}, 1\right) \text { if } B(x) \neq 0 \\ 1 \text { otherwise }\end{array}\right.$ (Goguen)

We choose the Lukasiewicz t-norm

$\mathrm{T}(\mathrm{a}, \mathrm{b})=\max (\mathrm{a}+\mathrm{b}-1,0)$.

3) Measures of similitude

$\mathrm{r}\left(\mathrm{B}, \mathrm{B}^{\prime}\right)=\sup _{\mathrm{x} \in \mathrm{X}} \min \left(\mathrm{B}(\mathrm{x}), \mathrm{B}^{\prime}(\mathrm{x})\right)$

$\mathrm{s}\left(\mathrm{C}, \mathrm{C}^{\prime}\right)=\sup _{\mathrm{y} \in \mathbb{Y}} \min \left(\mathrm{C}(\mathrm{y}), \mathrm{C}^{\prime}(\mathrm{y})\right)$

$R$ and $S$ are defined by any equal thresholds $\rho=\sigma$ in $[0,1]$, for the following fuzzy implication :

$\mathrm{I}(\mathrm{x}, \mathrm{y})=\min (\mathrm{B}(\mathrm{x}), \mathrm{C}(\mathrm{y}))$ (Mamdani)

We choose the Zadeh $t$-norm $T(a, b)=\min (a, b)$.

4) Measures of similitude

$\mathrm{r}\left(\mathrm{B}, \mathrm{B}^{\prime}\right)=\sup _{\mathrm{x} \in \mathrm{X}}\left(\mathrm{B}(\mathrm{x}) \cdot \mathrm{B}^{\prime}(\mathrm{x})\right)$

$s\left(C, C^{\prime}\right)=\sup _{y \in \Psi}\left(C(y) \cdot C^{\prime}(y)\right)$ 
$R$ and $S$ are defined by any equal thresholds $\rho=\sigma$ in $[0,1]$, for the following fuzzy implication :

$\mathrm{I}(\mathrm{x}, \mathrm{y})=\mathrm{B}(\mathrm{x}) \cdot \mathrm{C}(\mathrm{y})$ (Larsen)

We choose the product $t$-norm $T(a, b)=\min (a, b)$.

This can be interpreted as follows. If there exist a rule "if $\mathrm{X}$ is $\mathrm{B}$ then $\mathrm{Y}$ is $\mathrm{C}$ ", such that $\mathrm{B}^{\prime}$ resembles $\mathrm{B}$ at least at the level $\rho$ in $[0,1]$, then the compositional rule of inference yields a result $C^{\prime}$ which resembles $C$ also at least at the same level $\rho$, and the resemblance between $C$ and $C^{\prime}$ is the same as the comparison between $B$ and $B^{\prime}$, since the measure of satisfiability or similitude which is involved is the same. This proves that the compositional rule of inference works in an analogical way.

In the case of Mamdani and Larsen implications, which do not correspond to extensions of the implication in classical logic, but are known to be useful in fuzzy control, we obtain the same property of equivalence between the use of the compositional rule of inference (well-known fuzzy control methodology) and an analogical scheme.

\section{Analogical scheme for gradual reasoning}

Gradual knowledge is very common in knowledge-based systems, generally expressed as rules of the form "the more $\mathrm{X}$ is $\mathrm{B}$, the more $\mathrm{Y}$ is $\mathrm{C}$ ", or "the more $\mathrm{X}$ is $\mathrm{B}$, the more $\mathrm{Y}$ is $C$ is certain". There exist several approaches to such graduality [7][9][10]. Here, we restrict ourselves to a graduality represented by linguistic modifiers [2][7], such as "really" or "relatively", for instance. Linguistic modifiers are useful to modulate the fuzzy values of attributes, by weakening or reinforcing the meaning of a given value, for instance "small" yielding "very small" or "relatively small".

A modifier $m$ modulates the characterization $B$ of $X$ by creating a new characterization $B^{\prime}=\mathrm{mB}$ with membership function obtained by means of a transformation $\mathrm{t}_{\mathrm{m}}$ as such that $\forall \mathrm{x} \in \mathrm{X} \quad \mathrm{B}^{\prime}(\mathrm{x})=\mathrm{t}_{\mathrm{m}}(\mathrm{B}(\mathrm{x}))$ [2]. We use for instance modifier $m_{\alpha}$ [7] defined as follows. Other definitions would give analogous results [8].

$$
\begin{aligned}
& \forall x \in X m_{\alpha} B(x)=B(x+\alpha) \text { if } x+\alpha \in X \\
& m_{\alpha} B(x)=B\left(X^{-}\right) \text {if } x+\alpha<X^{-} \\
& m_{\alpha} B(x)=B\left(X^{+}\right) \text {if } x+\alpha>X^{+}
\end{aligned}
$$

if $X$ is supposed to be ordered, with smallest element $\mathrm{X}^{-}$, greatest element $\mathrm{X}^{+}$. If we apply this modifier to fuzzy descriptions of $X$, constituting a fuzzy partition of $X$, we obtain two kinds of behavior, either reinforcing ("really") or weakening ("relatively"), according to $\alpha$ being positive or negative and to the position of $B$ in the ordered list of classes of the partition (Figure 2).

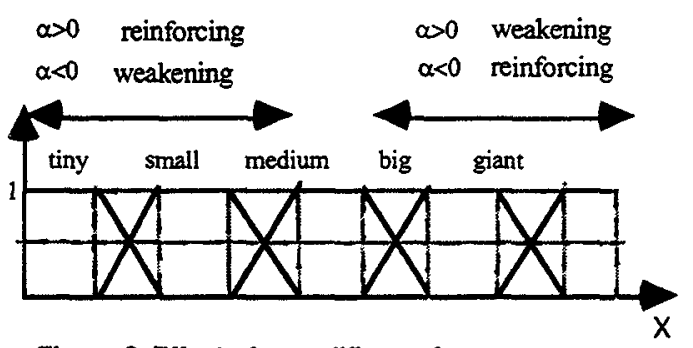

Figure 2. Effect of a modifier on fuzzy descriptions of $X$

The concept of graduality we use here corresponds to the fact that, if we weaken (or reinforce) the value of a variable $X$, then we weaken (or reinforce) also the value of a variable $\mathrm{Y}$ linked to $\mathrm{X}$. For instance "the more we separate the iris rhizomes, the more flowers we obtain". This is expressed as "if the value of $\mathrm{X}$ is reinforced, then the value of $Y$ is reinforced". It corresponds to the idea that a rule "if $\mathrm{X}$ is $\mathrm{m}_{\alpha} \mathrm{B}$, then $\mathrm{Y}$ is $\mathrm{m}_{\alpha}, \mathrm{C}$ " stems from a rule "is $\mathrm{X}$ is $\mathrm{A}$ then $Y$ is $B$ "

The graduality is defined by the fact that :

- the same modifiers (with linked values of parameters) are used for the descriptions of both variables,

- variations regarding membership functions are equal, i.e. :

$\forall \mathrm{x} \in \mathrm{X} \quad \forall \mathrm{y} \in \mathrm{X}(\mathrm{C}(\mathrm{y})=\mathrm{B}(\mathrm{x})) \Rightarrow$

$\left(\mathrm{m}_{\alpha}, \mathrm{C}_{\gamma}(\mathrm{y})-\mathrm{C}_{\gamma}(\mathrm{y})=\mathrm{m}_{\alpha} \mathrm{B}_{\gamma}(\mathrm{x})-\mathrm{B}_{\gamma}(\mathrm{x})\right)$

where $\gamma$ indicates the right or left hand part of the function.

We consider respective finite subsets $F(X)$ and $F(Y)$ of $[0,1]^{X}$ and $[0,1]^{Y}$ and $\left(R_{j}\right)$ a base of rules of the form "if $X$ is $B_{j}$ then $Y$ is $C_{j}$ ", with $B_{j}$ in $F(X)$ and $C_{j}$ in $F(Y)$, $j \in J, B_{j}$ and $C_{j}$ normalized fuzzy sets with trapezoidal membership functions.

The link $\beta$ is defined by :

$B \beta C \Leftrightarrow$ i) or ii) holds with :

i) $\exists$ an object in $E$ with value of $X$ equal to $B$ and value of $Y$ equal to $\mathrm{C}$

ii) $\exists R_{j}$ such that $B=B_{j}$ and $C=C_{j}$

We consider two operations defined by the inverse of the addition of fuzzy intervals, respectively on $X$ and $Y$, denoted by $r:[0,1]^{\mathbf{X}} \times[0,1]^{\mathbf{X}} \rightarrow[0,1]^{\mathrm{X}}$ and $s:[0,1]^{Y} \times[0,1]^{Y} \rightarrow[0,1]^{Y}$. The relations $R$ and $S$ are defined from $r$ and $s$ in such a way that there exist two thresholds $\rho \in[0,1]^{X}$ and $\sigma \in[0,1]^{Y}$ satisfying :

$\forall \mathrm{B} \forall \mathrm{B}^{\prime} \in[0,1]^{\mathrm{X}} \quad \mathrm{BRB}^{\prime} \Leftrightarrow \mathrm{r}\left(\mathrm{B}, \mathrm{B}^{\prime}\right)=\rho, \rho \in[0,1]^{\mathrm{X}}$

$\forall \mathrm{C} \forall \mathrm{C}^{\prime} \in[0,1]^{\mathrm{Y}} \quad \mathrm{CSC}^{\prime} \Leftrightarrow \mathrm{s}\left(\mathrm{C}, \mathrm{C}^{\prime}\right)=\sigma, \sigma \in[0,1]^{\mathrm{Y}}$,

We define a function $\mathfrak{R}_{\beta R S}$ as follows : 
$\Re_{\beta R S}\left(B, C, B^{\prime}\right)=C^{\prime} \Leftrightarrow B^{\prime}=m_{\alpha} B, C^{\prime}=m_{\alpha^{\prime}} C$,

with $\alpha=\phi(\rho), \sigma$ is obtained from $\alpha$ and from the difference between support and kernel of $B$ and $C$ [8], and $\alpha^{\prime}=\phi(\sigma)$.

It can be proven that $\mathfrak{R}_{\beta R S}$ is an analogical scheme.

This result can be extended to other kinds of modifiers. It expresses the fact that gradual reasoning can be regarded as a progressive passage from a reference given in a rule and other rules obtained from this reference by using linguistic modifiers. The link between gradual reasoning and analogical reasoning corresponds to the utilization of a relationship between variations of $X$ and variations of $Y$ expressed in gradual knowledge to infer a value of $Y$ from a given value of $X$. The links between this kind of graduality and interpolation [8] would lead to another form of analogical scheme for interpolative reasoning.

\section{Conclusion}

We have presented a general definition of analogy through a fonction considered as an analogical scheme, depending on a link between variables and relations generally defined from measures of proximity, resemblance, satisfiability and, more generally measures of comparison. This definition shows that several forms of reasoning in a fuzzy environment are based on the same approach and work in an analogical way. We will explore several other forms of reasoning from this point of view, such as interpolative reasoning, analogical reasoning, casebased reasoning...

\section{References}

[1] S. Bothorel. Analyse d'image par arbre de décision flou. Application à la classification des amas de microcalcifications. Thèse de l'Université P. et M. Curie, Paris, France, 1996.

[2] B. Bouchon-Meunier. Fuzzy logic and knowledge representation using linguistic modifiers, in Fuzzy logic for the management of uncertainty (L.A. Zadeh, J. Kacprzyk, eds.), John Wiley \& Sons, 1992, pp. 399-414.

[3] B. Bouchon-Meunier, C. Marsala and M. Rifqi. Comment classer des objets imparfaitement décrits? $X X V$ - colloque international de l'ARAE, Lyon, France, 1996.

[4] B. Bouchon-Meunier, C. Marsala and M. Ramdani. Learning from imperfect data. In H. Prade, D. Dubois and R.R. Yager eds, Fuzzy Information Engineering: a Guided Tour of Applications, pages 139-148, John Wileys and Sons, 1997.

[5] B. Bouchon-Meunier, M. Rifqi, S. Bothorel. Towards general measures of comparison of objects, Fuzzy Sets and Systems 84,2, pp. 143-153, 1996.

[6] B. Bouchon-Meunier, L. Valverde. Analogical Reasoning and Fuzzy Resemblance, in Uncertainty in Intelligent Systems (B. Bouchon-Meunier, L. Valverde, R.Yager, eds.), Elsevier Science Pub, pp 247-255, 1993.
[7] B. Bouchon-Meunier and J. Yao. Linguistic modifiers and gradual membership to a category, International Journal of Intelligent Systems 7, pp 25-36, 1992.

[8] J. Delechamp, B. Bouchon-Meunier, Graduality by means of analogical reasoning, Proc. European Conference ECSQARU, 1997, Springer Verlag, to appear

[9] D. Dubois, H. Prade. Gradual inference rules in approximate reasoning, Information Sciences, 1990.

[10] D. Dubois, H. Prade. Fuzzy rules in knowledge-based systems -Modelling gradeness, uncertainty and preference-, in An introduction to fuzzy logic applications in intelligent systems, (R.R. Yager, L.A. Zadeh, eds.) Kluwer Academic Publishers, 1992.

[11] C. Z. Janikow. Fuzzy decision trees: FIDMV. In JCIS'94, Proc. of the Joint Conference on Information Sciences, pages 232-235, Pinehurst, 1994.

[12] C. Marsala, B. Bouchon-Meunier, Forests of Fuzzy Decision Trees, In Proc. of the IFSA'97 World Congres, to appear, 1997.

[13] J. R. Quinlan, Induction of Decision Trees. Machine Learning, vol. $1, \mathrm{n}^{\circ} 1$, pages 86-106, 1986.

[14] M. Ramdani. Une approche floue pour traiter les valeurs numériques en apprentissage. In Journées francophones d'apprentissage et d'explication des connaissances, 1992

[15] M. Rifqi. Constructing prototypes from large databases, Proc. International Conference IPMU'96, Granada, 1996, pp. 301-306.

[16] M. Rifqi. Mesures de comparaison, typicalité et classification d'objets flous : théorie et pratique, Thèse de l'Université Paris VI, also Rapport LAFORIA TH96/15, 1996.

[17] E. Rosch. Principles of categorization in Cognition and categorization, E. Rosch and B. B. Lloyd editors, pages 27-48, 1978.

[18] I. B. Turksen, Zhao Zhong, An approximate analogical reasoning approach based on similarity measures, IEEE Trans. on Systems, Man and Cybernetics 18, 6, 1988.

[19] R. Weber. Fuzzy-ID3: a class of methods for automatic knowledge acquisition. In IIZUKA'92, Proc. of the 2nd Int. Conf. on Fuzzy Logic, vol. 1, pages 265-268, 1992.

[20] Y. Yuan and M. J. Shaw. Induction of fuzzy decision trees. Fuzzy Sets and Systems, $n^{\circ} 69$, pages 125-139, 1995.

[21] L. A. Zadeh. A note on prototype theory and fuzzy sets. Cognition, 12:291-297, 1982.

[22] L. A. Zadeh. The role of fuzzy logic in the management of uncertainty in Expert Systems, Fuzzy Sets and Systems 11, pp. 199-227, 1983. 